\title{
In vitro and in vivo biolasing of fluorescent proteins suspended in liquid microdroplet cavities $\dagger$
}

\author{
Alexandr Jonáš, ${ }^{* a}$ Mehdi Aas, ${ }^{b}$ Yasin Karadag, ${ }^{b}$ Selen Manioğlu, ${ }^{c}$ Suman Anand, ${ }^{d}$ \\ David McGloin, ${ }^{d}$ Halil Bayraktar ${ }^{c}$ and Alper Kiraz ${ }^{b}$
}

\begin{abstract}
Fluorescent proteins are indispensable for selective, quantitative visualization of localization, dynamics, and interactions of key molecular constituents of live cells. Incorporation of fluorescent proteins into an optical cavity can lead to a significant increase in fluorescence signal levels due to stimulated emission and light amplification in the cavity, forming a laser with biological gain medium. Utilization of lasing emission from fluorescent biological molecules can then greatly enhance the performance of fluorescence-based biosensors benefiting from the high sensitivity of non-linear lasing processes to small perturbations in the cavity and the gain medium. Here we study optofluidic biolasers that exploit active liquid optical resonators formed by surface-supported aqueous microdroplets containing purified yellow fluorescent protein or a suspension of live $E$. coli bacterial cells expressing the fluorescent protein. We first demonstrate lasing in fluorescent protein solutions at concentrations as low as $49 \mu \mathrm{M}$. Subsequently, we show that a single fluorescent bacterial cell of micrometre size confined in a droplet-based cavity can serve as a laser gain medium. Aqueous droplet microcavities allow the maintenance of the bacterial cells under conditions compatible with unimpeded growth. Therefore, our results also suggest a direct route to microscopic sources of laser light with self-regenerating gain media.
\end{abstract}

Received 24th April 2014, Accepted 12th June 2014

DOI: $10.1039 / c 4 l c 00485 j$

www.rsc.org/loc selective and sensitive quantitative detection of molecular components in complex environments (e.g., cell cytoplasm and membranes), visualization of protein expression and degradation in cells, and dynamics of molecular interactions. ${ }^{3}$

In a typical live-cell experiment, the intensity of spontaneous light emission from optically pumped fluorescent proteins expressed in cells is detected and evaluated. While this procedure is straightforward to implement, the level of the spontaneous fluorescence signal can be too low to collect a sufficiently high number of photons before the fluorophore photobleaches. Amplified stimulated emission from fluorescent protein molecules placed inside a suitable optical cavity can lead to lasing of the protein and, subsequently, a significant increase in the fluorescence intensity. Such biolasers can be used to implement new ways of biological sensing and analysis that benefit from the high sensitivity of stimulated emission to small perturbations in the laser cavity and the gain medium and from the specificity of attachment of fluorescent proteins to selected cellular components which allows targeted studies of well-defined intracellular processes. ${ }^{4}$

Biolasers can be readily integrated with existing optofluidic technologies; they can be combined with accurate control of fluids on small spatial scales and interfaced with optical waveguides or fibres in compact lab-on-a-chip systems. Gain media based on fluorescent proteins expressed in living 
cells can be regenerated following photobleaching of the fluorophore; hence, biolasers are in principle self-healing. Furthermore, specific molecular recognition and selfassembly can provide additional functionality and programmatic control of photonic components based on biological molecules. The first demonstrations of biolasing employed GFP-expressing live mammalian cells or $E$. coli bacteria placed inside a Fabry-Perot resonator. ${ }^{5,6}$ Recently, an optofluidic ring resonator (OFRR) was used for observation of in vitro lasing from aqueous solutions of fluorescent proteins. This scheme was based on indirect pumping of lasing emission via FRET and employed genetically encoded fluorescent protein pairs linked by a peptide with adjustable length, thus allowing the study of protein-protein or protein-drug interactions. ${ }^{7}$ Biological molecules other than fluorescent proteins have also been recently tested as laser gain media. In these demonstrations, lasing was achieved with luciferin solution contained within an $\mathrm{OFRR}^{8}$ or flavin mononucleotide dissolved in glycerol-water microdroplets supported by a superhydrophobic surface. ${ }^{9}$ However, unlike fluorescent proteins, these small fluorescent organic compounds cannot be readily expressed and regenerated in living cells and selectively fused to other cellular proteins without external coupling agents.

In this article, we introduce optofluidic droplet-based biolasers with the gain medium effectively formed by an individual micron-sized bacterial cell suspended in the droplet liquid. The glycerol-water microdroplets supported by a superhydrophobic surface serve as optical cavities for the amplification of stimulated emission from the Venus variant of yellow fluorescent protein expressed in the bacteria. Liquid optical resonators based on surface-supported aqueous microdroplets combine key advantages of OFRRs and FabryPerot resonators. While the optical modes of a Fabry-Perot resonator occupy relatively large mode volumes, OFRRs and spherical microdroplets host whispering gallery modes (WGMs) with very small mode volumes and high-quality factors (Q-factors). ${ }^{10}$ These morphology-dependent optical resonances circulate in a narrow region near the internal surface of the cavity due to the total internal reflection of light. As a result, very large optical intensities can be stored in WGMs. In an OFRR, however, the gain medium lies outside the cavity formed by the wall of a glass capillary tube, and thus, the gain medium only couples to the evanescent field of the WGMs circulating in the capillary wall. Hence, only the molecules within a narrow zone in the proximity of the capillary wall (less than $100 \mathrm{~nm}$ ) can undergo stimulated emission, which makes lasing from fluorescent proteins expressed in live cells very difficult. In contrast, in both Fabry-Perot cavities and microdroplet resonators, optical modes circulate directly in the volume where the gain medium is located, which implies a strong interaction between the light and the gain medium. The above combination of characteristic features renders microdroplet-based resonators very suitable for non-linear optical studies such as biolasing using low optical pump intensities. In addition, aqueous microdroplets provide natural environmental conditions for biological molecules and live cells. In our experiments, we characterize lasing from surface-supported droplets containing either solutions of purified Venus fluorescent protein or dilute suspensions of $E$. coli bacterial cells stably expressing the Venus protein. We study the dynamics of lasing from these laser gain media and provide experimental evidence for lasing from a single Venus-expressing bacterial cell.

\section{Materials and methods}

\subsection{Expression, isolation, and purification of Venus yellow fluorescent protein}

All reagents were obtained from VWR International GmbH (Darmstadt, Germany) and used as received unless otherwise specified. In order to express Venus in BL21 E. coli cells, the Venus gene was cloned into the pBTR $\left(\mathrm{Amp}^{\mathrm{r}}\right)$ expression vector using standard cloning methods and the plasmid was subsequently inserted into the bacteria. After transformation, BL21 cells were cultivated in $1 \mathrm{~L}$ of LB-type growth medium for 1 hour at $37^{\circ} \mathrm{C}$. Grown cells were harvested from the medium by centrifugation for 10 minutes at $4500 \mathrm{rpm}$ and then resuspended in $50 \mathrm{mM}$ phosphate buffer at $\mathrm{pH} 7$. Cells in buffered suspensions were homogenized with a probe-tip sonicator. The resulting cell lysate was then ultra-centrifuged at $20000 \mathrm{rpm}$ for 15 minutes and the supernatant was collected and used in all lasing experiments. The emission and excitation fluorescence spectra of $5 \mu \mathrm{M}$ Venus solution in $50 \mathrm{mM}$ phosphate buffer were measured at $25^{\circ} \mathrm{C}$ with a FluoroMax-3 spectrofluorometer. The absorption spectra of the suspended Venus-expressing BL21 cells were collected using a Shimadzu UV-3600 spectrophotometer. For quantitative analysis of the fluorescence intensity of purified Venus solutions and Venus-expressing $E$. coli cells, the samples were imaged with an EMCCD camera (Lucas, Andor) operated in frame-transfer mode at 5 frames per second. Fluorescence emission data were analysed using Image (NIH) and MATLAB (MathWorks) software packages.

\subsection{Generation of liquid microdroplets supported by superhydrophobic surfaces}

Superhydrophobic surfaces with water contact angles $>150^{\circ}$ for droplet deposition were prepared by spin coating hydrophobic silica nanoparticles (Aeroxide LE2, Evonik) dispersed in ethanol on cleaned coverslips. ${ }^{11}$ To deposit micron-sized droplets on the surface, liquid aerosols containing the laser gain medium in the form of either purified Venus protein or fluorescent bacteria in a mixture of water and glycerol were generated using a compact ultrasonic nebulizer (JIH50, Beuer) and sprayed over the surface. This procedure provided surface-supported microdroplets with radii ranging from $0.5 \mu \mathrm{m}$ to $50 \mu \mathrm{m}$; in the case of bacterial suspensions, the mixture was diluted until the droplets contained approximately $5-15$ bacteria per droplet with a radius of $\sim 5-10 \mu \mathrm{m}$. The number of bacteria in the selected droplet was estimated by direct counting of the suspended cells. In order to obtain 
the in-focus image of the cells, the position of the microscope focal plane within the droplet was gradually adjusted during counting.

\subsection{Fluorescence spectroscopy of lasing microdroplets}

A detailed scheme of the inverted optical microscope system used for recording the emission spectra of the surfacesupported fluorescent microdroplets is shown in Fig. S1 in the ESI. $\dagger$ An individual selected droplet resting on the superhydrophobic surface was optically pumped with a pulsed femtosecond $488 \mathrm{~nm}$ laser beam (pulse width $140 \mathrm{fs}$, repetition rate $80 \mathrm{MHz}$; Chameleon Ultra II, Coherent) in $5 \mu \mathrm{s}$ bunches of pump pulses (approximately 400 pulses per bunch; see the ESI $\dagger$ Fig. S2). In order to minimize the distortion of the studied droplet shape by intense laser pulses, a single bunch of excitation pulses was used for recording the emission spectrum of the droplet. The excitation beam was focused at the rim of the studied droplet using a high-NA water-immersion microscope objective lens $(60 \times, \mathrm{NA}=1.2$; Nikon) to a diffraction-limited spot with a radius of approximately $0.4 \mu \mathrm{m}$. Fluorescence emission from the droplets was then collected using the same microscope objective and analysed with an imaging spectrometer.

\section{Results}

\subsection{Characterization of Venus fluorescent protein and} Venus-expressing bacterial cells

Venus yellow fluorescent protein has been frequently used as a reporter for the quantification of protein expression levels. ${ }^{12-16}$ Since Venus has a high quantum yield of $\Phi=0.57$ (ref. 17) and it is one of the brightest fluorophores among all fluorescent proteins ${ }^{18}$ it is an excellent candidate for the gain medium of optofluidic biolasers. In our experiments, Venus was expressed in BL21 E. coli cells using a pBTR highexpression vector. The resulting high intracellular concentration of the fluorophore is a prerequisite for observation of live-cell lasing emission with a low pump laser power. In Fig. 1a, an aqueous suspension of Venus-expressing BL21 bacterial cells is shown under white light illumination (left) and UV light illumination (right). In both imaging modes, the bacterial suspension displays an intense colour (yellow for the transmitted white light image, light green for the fluorescence image) indicative of a high Venus expression level in BL21 cells. Individual fluorescent bacteria deposited on a cover slip surface could be clearly imaged under wide-field illumination with $473 \mathrm{~nm}$ laser light (Fig. 1b). To determine the maximum absorption wavelength of Venus under in vivo conditions, we acquired the visible absorption spectrum of an aqueous suspension of fluorescent BL21 cells at $\mathrm{pH} 7.0$ (Fig. 1c). In this spectrum, the main absorption peak of intracellular Venus at $515 \mathrm{~nm}$ is superimposed on the monotonically decreasing spectral background caused by light scattering from the cells. The spectrum also features a secondary absorption shoulder around $488 \mathrm{~nm}$, which was used in the Venus lasing experiments. Further information about the a
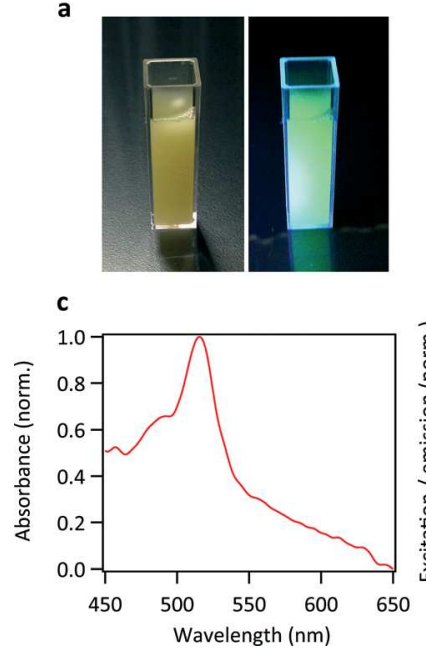

b
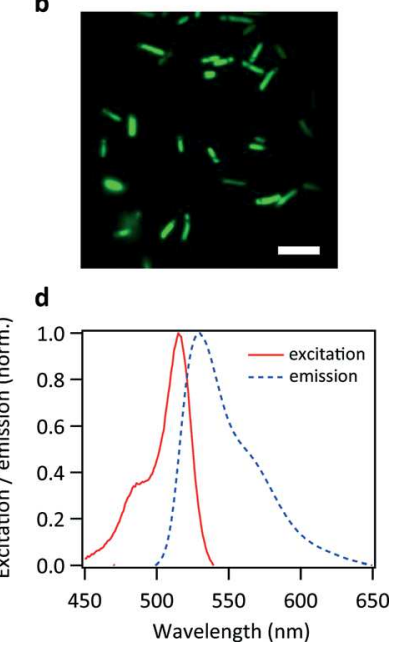

Fig. 1 Venus-expressing $E$. coli cells as the laser gain medium. (a) The image of an aqueous suspension of the BL21 strain of $E$. coli bacterial cells expressing the Venus variant of the yellow fluorescent protein under white light illumination (left) and UV light illumination (right). (b) The pseudocolor fluorescence image of BL21 cells expressing Venus fluorescent protein. The scale bar is $5 \mu \mathrm{m}$. (c) The absorption spectrum of an aqueous suspension of BL21 cells expressing Venus fluorescent protein. (d) Excitation and emission spectra of the purified Venus fluorescent protein suspended in $50 \mathrm{mM}$ phosphate buffer.

spectral properties of Venus was obtained from the fluorescence excitation and emission spectra of the solutions of purified Venus in $50 \mathrm{mM}$ phosphate buffer (Fig. 1d). The concentration of Venus in the buffer solution $(5 \mu \mathrm{M})$ was determined from its recorded visible absorption spectrum and known molar extinction coefficient. ${ }^{12}$ The spectral peak of Venus fluorescence emission recorded with $470 \mathrm{~nm}$ excitation light was observed at $530 \mathrm{~nm}$. Identical to the live-cell absorption spectrum of Fig. 1c, the fluorescence excitation spectrum displays a maximum at $515 \mathrm{~nm}$. This suggests that the high expression level of Venus in the bacteria does not have a significant influence on the spectral characteristics and the fluorescence brightness of the protein. In order to estimate the concentration of Venus in living BL21 cells, we compared the intensity of fluorescence from individual cells and a Venus solution of a known protein concentration (see the ESI $\dagger$ text and Fig. S3). The average intracellular concentration of Venus, $c_{\mathrm{B}}$, was found to be approximately $470 \mu \mathrm{M}$. Considering a typical $E$. coli cell volume, $V_{\mathrm{B}}$, of $0.20-0.77 \mathrm{fl}$ (cell volume approximated by a cylinder with a diameter of 0.5-0.7 $\mu \mathrm{m}$ and a length of 1-2 $\mu \mathrm{m}$ ), this corresponds to $N_{\mathrm{A}} V_{\mathrm{B}} c_{\mathrm{B}} \approx 60000-220000$ copies of Venus per cell $\left(N_{\mathrm{A}}\right.$ is Avogadro's number).

\subsection{Observation of lasing from aqueous solutions of Venus fluorescent protein}

Liquid microdroplets supported by superhydrophobic ( $\mathrm{SH}$ ) surfaces have been successfully used as optical resonant cavities in the studies of lasing from aqueous solutions of fluorescent dyes ${ }^{11}$ and vitamins. ${ }^{9}$ Deposition of droplets on an 
SH surface with a high liquid-solid contact angle preserves the spherical shape of the droplets and stabilizes their position. Moreover, with transparent droplet-supporting substrates, light can be easily coupled into and out of the droplets using an inverted optical microscope (Fig. 2a). In our experiments, we obtained transparent SH surfaces by spincoating a solution of hydrophobic silica nanoparticles on clean glass coverslips. The studies of lasing from aqueous solutions of Venus fluorescent protein were carried out using Venus dissolved in a $35 \%(\mathrm{v} / \mathrm{v})$ mixture of glycerol and $50 \mathrm{mM}$ phosphate buffer. The presence of low-volatility glycerol in the droplet liquid is essential for stabilizing the droplets exposed to an ambient room atmosphere against evaporation. ${ }^{19}$ Under typical experimental conditions (temperature $26{ }^{\circ} \mathrm{C}$, relative humidity $64 \%$ ), the final equilibrium concentration of glycerol in the phosphate buffer solution was estimated to be approximately $64 \%(\mathrm{v} / \mathrm{v})$; this corresponds to the
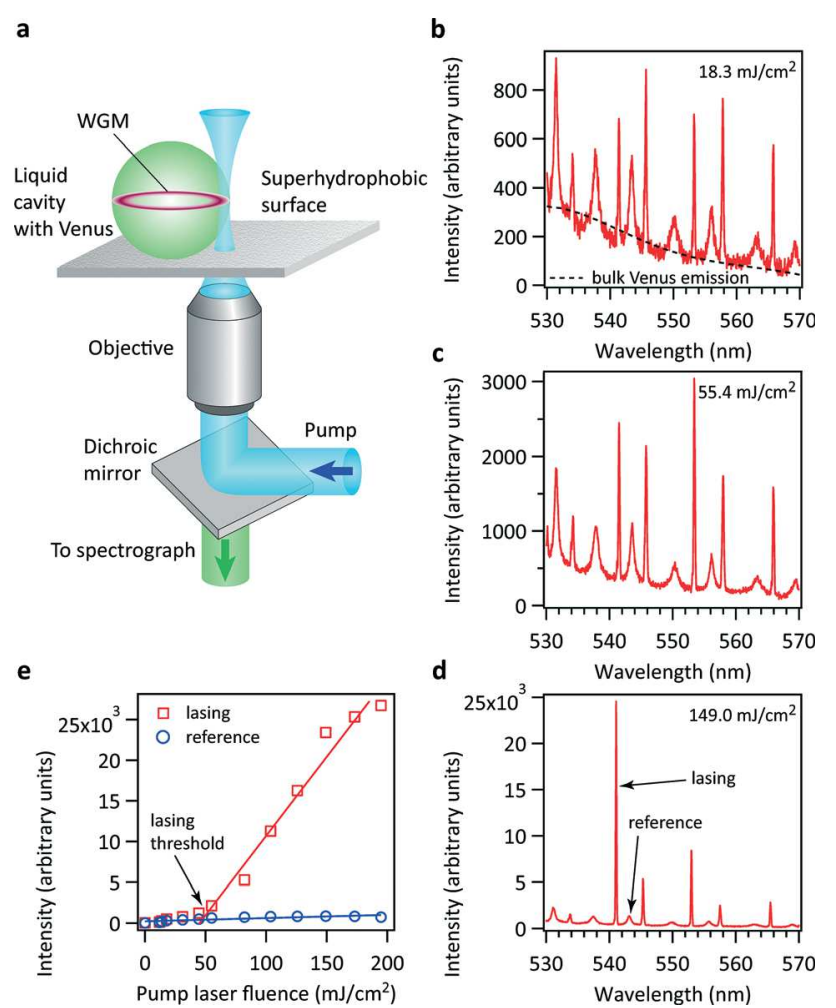

Fig. 2 Lasing of Venus fluorescent protein in surface-supported microdroplets. (a) Experimental geometry for observation of lasing from the solution of purified Venus fluorescent protein contained in a surface-supported glycerol-water microdroplet acting as a liquid optical resonant cavity. The figure is not drawn to scale. (b) An example of the droplet emission spectrum recorded with a pump beam fluence below the lasing threshold. The black dashed line shows the bulk emission spectrum of Venus fluorescent protein solution (no cavity modes). (c) An example of the droplet emission spectrum recorded with a pump beam fluence near the lasing threshold. (d) An example of the droplet emission spectrum recorded with a pump beam fluence above the lasing threshold. (e) The intensity of the lasing and the reference spectral peaks indicated in (d) as a function of the pump laser fluence. Symbols denote experimental data; solid lines denote linear fits to the data. The droplet radius is $3.2 \mu \mathrm{m}$. reduction of the droplet volume by a factor of 0.55 and, consequently, the increase in the Venus concentration in the droplets by a factor of 1.81. All concentrations of Venus in droplets reported in this article are the final values that take into account partial liquid evaporation after the droplet generation. Venus-containing liquid droplets were deposited on the SH surface using a compact ultrasonic nebulizer. Subsequently, fluorescence from individual droplets was excited by focusing a femtosecond (fs) pulsed laser beam with a wavelength of $488 \mathrm{~nm}$ at the droplet rim using a microscope objective of high numerical aperture. Light emitted from the droplet was collected using the same objective lens and analysed using a spectrograph (see the ESI† text and Fig. S1 for details).

In Fig. 2b-d, the emission spectra are presented which were recorded from a single surface-supported droplet with a Venus concentration of $49 \mu \mathrm{M}$ and consecutively increasing fluence of the pump laser beam. At the lowest excitation fluence of $18.3 \mathrm{~mJ} \mathrm{~cm}^{-2}$ (Fig. 2b), the pump energy is not sufficient to achieve lasing for the given Venus concentration. The resulting droplet emission spectrum features a number of non-lasing WGMs of comparable intensity and varying width superimposed on a broad non-resonant background of bulk Venus fluorescence. The observed WGMs correspond to different resonant paths of the light in the droplet and can be uniquely identified by their radial, angular, and azimuthal mode numbers and mode polarization (TE or TM). ${ }^{20}$ In general, WGMs with the lowest radial mode number $(n=1)$ that circulate closest to the droplet surface display the highest Q-factors; thus, they are the best candidates for the observation of lasing. ${ }^{21}$ When the pump fluence increases to $55.4 \mathrm{~mJ} \mathrm{~cm} \mathrm{~cm}^{-2}$, the intensity of these narrowest WGMs increases by a larger factor compared to the intensity of broad WGMs and the non-resonant spectral background (Fig. 2c). The stronger dependence of the intensity of high-Q WGMs on the pump fluence indicates the onset of nonlinear optical phenomena associated with amplified stimulated emission from the droplets. With the pump fluence set to $149 \mathrm{~mJ} \mathrm{~cm}^{-2}$, well above the lasing threshold of the studied droplet, the intensity of lasing WGMs relative to non-lasing optical modes and the non-resonant background increases dramatically (Fig. 2d). The spectral location of the overall maximum of lasing emission is then determined by the interplay between the Venus absorption and emission profiles, Venus concentration in the droplet, and droplet size. Generally, the strongest lasing peak is shifted to the red from the bulk emission maximum of the fluorophore due to the self-absorption that increases absorptive losses of the cavity at shorter emission wavelengths. ${ }^{22}$

In order to quantify the pump threshold required for droplet lasing, we recorded and analysed a series of droplet emission spectra with gradually increasing fluence of the pump beam. In Fig. 2e, the intensity above background for lasing and reference WGMs annotated in Fig. $2 \mathrm{~d}$ is plotted as a function of the pump fluence. From this figure, it is obvious that upon exceeding a certain pump level, intensities of 
the lasing and reference WGMs start increasing at a different rate, which marks the onset of lasing. An actual threshold value of $44.5 \mathrm{~mJ} \mathrm{~cm}^{-2}$ was determined from the intersection of line fits to the experimental data points obtained for the reference WGM (over the whole range of studied pump fluences) and the lasing WGM (pump fluences above $46 \mathrm{~mJ} \mathrm{~cm}^{-2}$ ). After crossing the threshold, the full width at half maximum (FWHM) of the reference WGM remains more or less constant $(\sim 0.65 \mathrm{~nm})$, whereas the FWHM of the lasing WGM decreases by approximately $20 \%$ from $0.22 \mathrm{~nm}$ to $0.18 \mathrm{~nm}$ (see the ESI $\dagger$ Fig. S4) due to the nonlinear interactions between the amplified resonant light and the molecules of the gain medium. ${ }^{11}$ Similar to regular fluorescent dyes, the pump threshold of the Venus-based microdroplet lasers is a function of the protein concentration in the droplets and the droplet radius, as both of these factors influence the overall losses of the cavity. ${ }^{22}$

\subsection{Stability of lasing from aqueous Venus solutions}

Since the total amount of Venus fluorescent protein contained in any given droplet is constant, the effective lifetime of droplet-based microlasers using Venus as the gain medium is limited by the rate of protein photobleaching. In order to characterize the Venus photobleaching dynamics, we recorded a time series of lasing spectra from individual Venuscontaining droplets using a constant fluence of the pump beam. Fig. 3a displays a typical series of emission spectra acquired from a $2.4 \mu \mathrm{m}$ radius droplet containing $49 \mu \mathrm{M}$ Venus solution. The effective acquisition time for each spectrum was $\sim 5 \mu \mathrm{s}$, corresponding to the duration of the bunch of excitation pulses (see the ESI $\uparrow$ Fig. S2), and the delay between individual spectral frames was $1 \mathrm{~s}$. A constant excitation fluence of $246 \mathrm{~mJ} \mathrm{~cm}^{-2}$ was set to be above the initial lasing threshold that characterizes the droplets before any Venus photobleaching has taken place. In Fig. 3a, the WGM at $533 \mathrm{~nm}$ labelled "lasing" is amplified via stimulated emission within the droplet cavity, whereas the WGM at $539 \mathrm{~nm}$ labelled "reference" represents regular spontaneous emission. These two WGMs belong to different mode families with
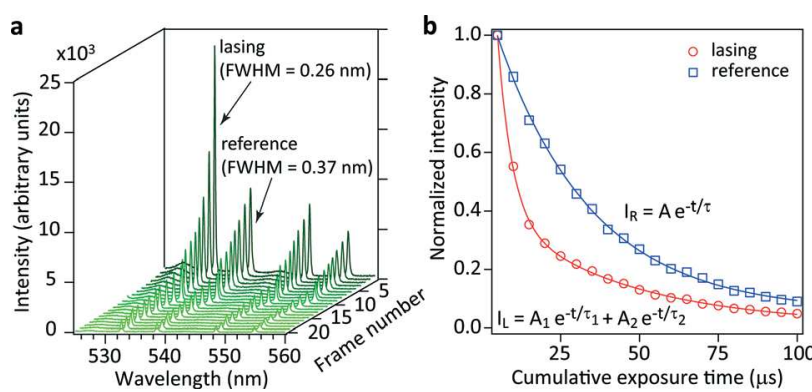

Fig. 3 Stability of lasing from Venus protein solutions. (a) Time series of the lasing emission spectra recorded with a constant pump fluence from a surface-supported microdroplet with a radius of $2.4 \mu \mathrm{m}$. (b) Time evolution of the normalized intensity of lasing and reference WGMs denoted in (a). Symbols indicate experimental data points; solid lines indicate double-exponential (lasing WGM intensity, $I_{L}$ ) or singleexponential (reference WGM intensity, $I_{\mathrm{R}}$ ) fits to the experimental data. identical radial mode numbers and different polarizations: TM for the lasing WGM and TE for the reference WGM. ${ }^{23}$ The lasing WGM has a smaller initial FWHM than the reference WGM $(0.26 \mathrm{~nm} v s .0 .37 \mathrm{~nm})$ which translates into a higher Q-factor of the lasing WGM. From Fig. 3a, it is obvious that the intensity of all WGMs in the spectrum monotonically decreases with increasing acquisition frame number due to increasing total exposure of the droplet to the pump beam and, consequently, higher Venus photobleaching.

More quantitative insight into the photobleaching processes can be obtained from the analysis of the time evolution of the WGM intensity presented in Fig. 3b. In this figure, normalized amplitudes above the spectral background of the lasing and reference WGMs are plotted as a function of the cumulative exposure time of the droplet to the pump beam. Here, the amplitudes of both modes are normalized to their respective initial values in the first recorded spectral frame. Comparison of the experimental data recorded for the lasing and reference WGMs reveals different dynamics of the intensity decay of both modes. While the intensity $I_{R}$ of the reference WGM decreases with a single-exponential decay of the form $I_{\mathrm{R}}=A \exp (-t / \tau)$, the intensity $I_{\mathrm{L}}$ of the lasing WGM cannot be described using a single-exponential decay, following instead a double-exponential decay of the form $I_{\mathrm{L}}=A_{1}$ $\exp \left(-t / \tau_{1}\right)+A_{2} \exp \left(-t / \tau_{2}\right)$; adding further exponential terms does not lead to a significant change in the fit residuals. The intensity of the remaining two WGMs and the spectral background decreases exponentially with the same characteristic time as the reference WGM. In homogeneous aqueous solutions of fluorescent molecules, the photobleaching reaction can be regarded as quasi-unimolecular and, thus, fluorophore concentration decreases exponentially with time. ${ }^{24}$ This notion agrees with the observed behaviour of the intensity of non-lasing WGMs and the non-resonant spectral background originating from spontaneous emission which depends linearly on the Venus concentration. On the other hand, Venus lasing involves non-linear optical processes that underlie amplified stimulated emission taking place in the dropletbased optical resonator. Overall cavity gain and losses depend on the actual Venus concentration in the cavity and their interplay determines the pump threshold and the intensity of the lasing peak. As the fluorescent protein progressively bleaches, the pump threshold required for lasing gradually increases until it exceeds the actual used pump fluence; at this point, the lasing action ceases. A double-exponential fit to the temporal profile of the lasing WGM intensity shown in Fig. $3 \mathrm{~b}$ gives two characteristic decay times, $\tau_{1}=4.5 \mu \mathrm{s}$ and $\tau_{2}=40.5 \mu \mathrm{s}$. On the fast time scale described by $\tau_{1}$, WGM emission is dominated by lasing that eventually changes to spontaneous emission when the slow time scale $\tau_{2}$ is reached. This interpretation is supported by the measured characteristic decay time $\tau$ of the reference WGM that is found to be $\tau=30 \mu \mathrm{s}$, comparable to $\tau_{2}$. The gradual decrease of the lasing contribution to the overall emission intensity is also accompanied by broadening of the lasing WGM whose FWHM increases to $0.36 \mathrm{~nm}$ after a cumulative exposure of $100 \mu \mathrm{s}$ 
and, thus, becomes comparable to the FWHM of the reference WGM (see the ESI† Fig. S5).

The lifetime of the first excited electronic state of Venus fluorescent protein from which stimulated emission to the ground electronic state occurs is approximately $3 \mathrm{~ns} .{ }^{25}$ The femtosecond pump laser used in our experiments has a pulse repetition rate of $80 \mathrm{MHz}$, which corresponds to a pulse-topulse separation of $12.5 \mathrm{~ns}$, longer than the fluorescence lifetime. Thus, lasing emission from the droplets can be excited with a single pump pulse. A typical spectral frame shown in Fig. 3a is acquired with $\sim 400$ pump pulses. Assuming $100 \%$ efficiency of the pumping, $\sim 400$ lasing pulses are emitted from the droplet in a single spectrum. Since lasing is typically observable in several consecutive spectral frames before Venus bleaches, the minimal useful output of droplet-based microlasers is a few thousands pulses.

\subsection{Observation of lasing in suspensions of bacteria expressing Venus fluorescent protein}

After characterization of the basic properties of droplet-based microlasers with aqueous solutions of purified Venus fluorescent protein, we carried out experiments in which the laser gain medium was formed by dilute suspensions of living BL21 E. coli bacteria stably expressing Venus. To this end, bacterial cells were suspended in a $35 \%(\mathrm{v} / \mathrm{v})$ mixture of glycerol and $50 \mathrm{mM}$ phosphate buffer (initial glycerol concentration prior to droplet generation and partial evaporation; see the discussion in section 3.2). Subsequently, liquid droplets were deposited on the SH surface using a nebulizer. Cell concentration in the suspension was adjusted so as to obtain approximately 5-15 bacteria per droplet with a radius of 5-10 $\mu \mathrm{m}$. In order to excite fluorescence in the droplets, the pump laser beam was focused at the droplet rim over a suitably positioned bacterial cell (see Fig. $4 \mathrm{a}$ and the inset of Fig. $4 \mathrm{~b}$ for details of the excitation geometry). Fig. $4 \mathrm{~b}$ shows an example
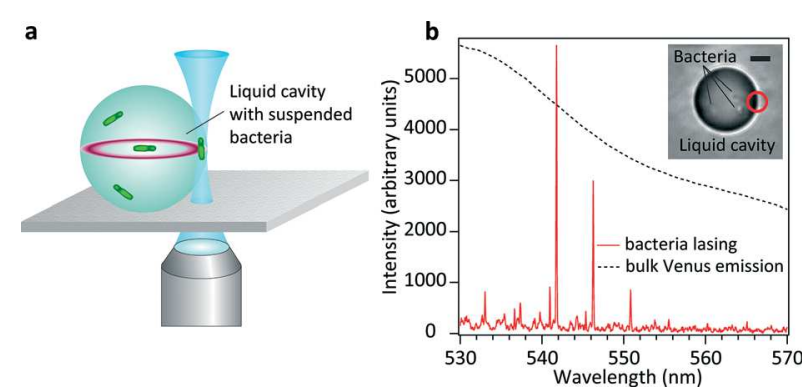

Fig. 4 Lasing of fluorescent $E$. coli bacterial cells in surface-supported microdroplets. (a) Experimental geometry for observation of lasing from live $E$. coli bacterial cells expressing Venus fluorescent protein. The bacteria are suspended and freely mobile in glycerol-water microdroplets standing on a superhydrophobic surface. The figure is not drawn to scale. (b) An example of the lasing emission spectrum recorded from suspended $E$. coli bacterial cells (solid line). For comparison, the emission spectrum of a purified bulk solution of Venus fluorescent protein is also shown (dashed black line). The inset is the image of the actual droplet (radius $8.4 \mu \mathrm{m}$ ) used for spectral recording that contains $\sim 7$ bacterial cells. The circle indicates the location of the excitation beam spot on the droplet. The scale bar is $5 \mu \mathrm{m}$. of the emission spectrum recorded from an $8.4 \mu \mathrm{m}$ radius droplet containing $\sim 7$ bacterial cells. The excitation fluence used for recording the spectrum was $312 \mathrm{~mJ} \mathrm{~cm}^{-2}$ and the effective acquisition time was $5 \mu \mathrm{s}$. Sharp lasing WGMs observed between 540-555 $\mathrm{nm}$ are shifted to the red with respect to the emission maximum of bulk Venus solution $(530 \mathrm{~nm})$ due to self-absorption induced losses at shorter emission wavelengths. The FWHM of lasing WGMs in the bacterial suspension $(0.13 \mathrm{~nm})$ is comparable to the FWHM of the lasing peak in Venus solution (0.18 $\mathrm{nm}$ - see Fig. 2d), which implies comparable Q-factors and, consequently, negligible distortion of the droplet shape and minimal scattering losses due to the presence of bacteria in the liquid.

Unlike in the case of droplets containing purified Venus solution, laser gain medium represented by individual fluorescent bacterial cells is discrete, with random and nonuniform distribution within the liquid cavity. Moreover, the cells can move freely in the liquid due to diffusion and active motion. The size of the effective excitation volume formed in the vicinity of the pump beam focus is comparable to the wavelength of the pump beam; thus, only a single bacterial cell typically resides within this high-intensity excitation region. An additional restriction on the permissible location of the lasing bacterium within the droplet arises from the requirement of a sufficient overlap of the bacterium with the WGM modal volume, which is a prerequisite for the observation of stimulated emission. As argued in section 3.2, lasing is associated with the highest-Q WGMs characterized by the lowest radial mode number $n=1$ (ref. 21). These WGMs are confined to radial distances $r$ from the droplet centre by $0.9 a<r<a$, where $a$ is the droplet radius. ${ }^{26}$ For the typical size of droplets used in our lasing experiments $(a<10 \mu \mathrm{m})$, the thickness of the spherical shell near the droplet surface, within which the stimulated emission can occur, lies in the sub-micron range. The limited spatial extent of the excitation region and the modal volume of WGMs together with the random motion of the bacteria into and out of the excitation volume lead to a distinctive on-off character of the lasing emission from dilute bacterial suspensions contained in the droplets. Fig. 5a presents a time series of fluorescence emission spectra recorded from a droplet with a $6.8-\mu \mathrm{m}$ radius containing approximately 10 bacterial cells. A constant pump fluence of $312 \mathrm{~mJ} \mathrm{~cm}^{-2}$ was used, with an effective integration time of $5 \mu$ s for acquiring each spectrum in the series, and a total of 32 spectra were recorded with a time delay of $1 \mathrm{~s}$ between successive spectra. In the 2-D spectrum of Fig. 5a, random frame-to-frame intensity fluctuations are clearly observable. In addition, small variations in the spectral positions of individual WGMs appear which may be attributed to changes in the WGM resonant path length induced by random diffusion of the cells through the mode path and fluctuations of the droplet cavity size caused by the interplay between the droplet evaporation and condensation during optical pumping. ${ }^{19}$ Four selected spectral frames highlighted by white horizontal lines in Fig. 5a and shown in detail in Fig. 5b-e illustrate the dynamics of the droplet 

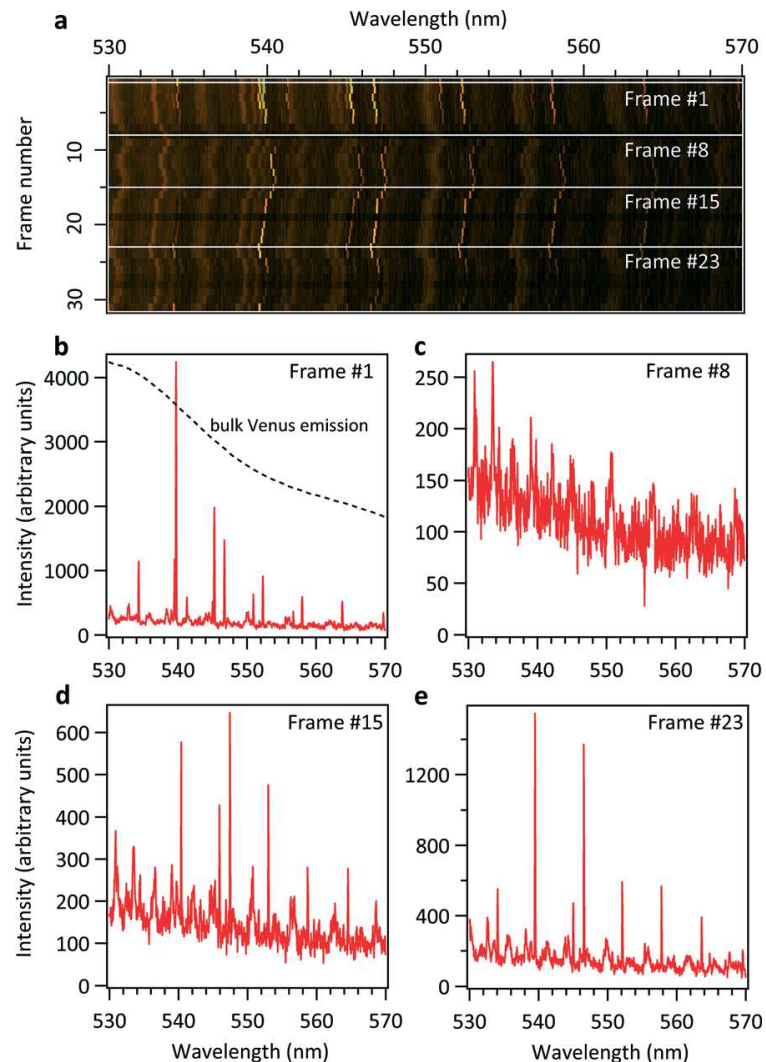

Fig. 5 Dynamics of lasing from suspended E. coli bacterial cells. (a) Time series of emission spectra recorded from a bacteriacontaining droplet of $6.8 \mu \mathrm{m}$ radius with a constant pump fluence. The number of bacterial cells in the droplet was $\sim 10$. (b-e) Droplet emission spectra for frames $1,8,15$, and 23 indicated by the white horizontal lines in (a). Due to bacteria diffusion and active motion into and out of the excitation volume, the droplet emission pattern in different frames switches between the lasing and the non-lasing emission mode.

emission pattern. From initial lasing in frame \#1, droplet emission progresses to an almost dark state with a very low emission intensity in frame $\# 8$ as the bacterium forming the laser gain medium tumbles in the liquid (see the ESI $\dagger$ Video 1 and Video 2 for illustration of bacterial motion). Subsequently, the bacterium approaches the excitation volume again, displaying regular non-lasing WGMs in frame \#15, and the droplet then returns back to lasing in frame \#23. During the random motion of the selected bacterium through the excitation volume, transitions from amplified stimulated emission to spontaneous emission are marked by large fluctuations of the WGM intensity. Moreover, frame-to-frame changes of the cell position and orientation also result in changes of the relative intensity of individual lasing WGMs since their relative gain and losses are highly sensitive to small perturbations in the liquid cavity.

\section{Discussion}

We have demonstrated and characterized lasing from active liquid optical microcavities formed by surface-supported water/glycerol microdroplets containing purified Venus fluorescent protein or dilute suspension of live $E$. coli bacterial cells expressing stably the Venus protein. Using aqueous Venus solutions, we have shown that lasing can be achieved with the Venus concentration in the droplet as low as a few tens of micromolars, which compares favourably with microdroplet lasers that exploit traditional organic dyes. ${ }^{11}$ Under femtosecond pulsed laser excitation, optofluidic biolasers based on Venus-containing microdroplets can emit several thousand pulses before the fluorophore photobleaches. As suggested by Eggeling et al., detrimental effects of photobleaching on the useful operation time of such droplet-based microlasers can be moderated by using excitation wavelengths closer to the Venus absorption maximum $(515 \mathrm{~nm})$ and longer excitation pulses. ${ }^{27}$ Alternatively, Venus can be replaced with a different fluorescent protein that displays a better photostability. ${ }^{17}$ Our studies of lasing in microdroplets prepared from dilute aqueous suspensions of fluorescent $E$. coli bacteria expressing Venus have provided experimental evidence for the feasibility of such biolasers with active gain medium formed by live cells suspended in a liquid under environmental conditions compatible with cell survival. The distinctive on-off character of lasing emission from bacterial suspensions supports the notion that a single fluorescent bacterium is sufficient as the laser gain medium. Irradiation of the selected bacterial cell serving as the laser gain medium with the high-fluence pump beam raises the question of cell viability upon prolonged light exposure. Even though we did not observe any obvious changes in the integrity of the irradiated cells after recording the spectra, we cannot rule out a more subtle damage to the vital organelles of the cell. Daddysman and Fecko studied the localized damage of DNA in eukaryotic cells induced by multi-photon absorption of focused femtosecond laser pulses with the pulse width, repetition rate, wavelength, and focal spot radius very similar to those of our experiments. ${ }^{28}$ They found out that a total energy of 100-300 nJ deposited to the specimen (HeLa cells or polytene nuclei of Drosophila salivary gland cells) is sufficient to cause observable DNA damage. In our experiments with bacteria lasing, the typical energy deposited to the lasing cell for recording a single spectral frame was approximately $600 \mathrm{~nJ}$ (mean power of $120 \mathrm{~mW}$ at the specimen with $5 \mu$ s exposure time). Thus, it is quite likely that the bacterial DNA was at least partially damaged. However, in our case, not all of the pump laser energy reaching the specimen was actually absorbed by the DNA. In E. coli, the nucleoid (the bacterial chromosome) typically occupies a region with a volume of about $0.23 \mu \mathrm{m}^{3}$ which corresponds to a sphere with a radius of approximately $0.38 \mu \mathrm{m},{ }^{29}$ equal to the radius of the pump beam waist used in our study $(\sim 0.40 \mu \mathrm{m})$. Since the Venus-expressing irradiated cells displayed strong fluorescence, it follows that a significant part of the pump light was absorbed by the fluorescent protein and, thus, it did not reach the DNA. Moreover, even if the originally selected lasing cell is irreversibly damaged by the pump beam, other cells within the same droplet that have not been exposed to the pump light are unaffected, and 
consequently, they can be used as the gain medium in the next trial.

\section{Conclusion}

We introduced and systematically studied miniature optofluidic biolasers based on surface-supported liquid microdroplet cavities containing either purified yellow fluorescent protein or live E. coli bacteria expressing this protein. Initially, we demonstrated that micromolar protein concentrations in aqueous microdroplets are sufficient for sustained lasing. Subsequently, we showed that a single micron-sized fluorescent bacterial cell can serve as living laser gain medium. The lasing bacteria suspended in a droplet can be provided with all nutrients required for steady growth, which may allow for extension of the operation time of this biologically controlled source of laser light by active regeneration of the gain medium. Using bacterial strains expressing two different fluorescent proteins that form a FRET donor-acceptor pair, a non-radiative energy-transfer mechanism can be used for laser pumping which would further improve lasing efficiency and open up the possibility for emission wavelength tuning. ${ }^{30}$ Furthermore, FRET lasing in connection with self-recognition and selfassembly of biological molecules attached to the fluorescent proteins can be exploited to program and modulate the laser characteristics, thus paving the way to new routes of live-cell bio-sensing.

\section{Acknowledgements}

We acknowledge financial support from TÜBITAK (grant no. 111T059). SA acknowledges COST Action MP1205 for support. DM acknowledges support from EPSRC (grant no. EP/G007713/1). The plasmid $\left(A m p^{r}\right)$ containing the Venus gene was kindly provided by Adam E. Cohen of Harvard University. We thank Melikhan Taniyeri for proofreading the manuscript.

\section{Notes and references}

1 S. J. Remington, Protein Sci., 2011, 20, 1509-1519.

2 J. Wiedenmann, F. Oswald and G. U. Nienhaus, IUBMB Life, 2009, 61, 1029-1042.

3 M. Zimmer, Chem. Rev., 2002, 102, 759-781.

4 X. Fan and S. H. Yun, Nat. Methods, 2014, 11, 141-147.

5 M. C. Gather and S. H. Yun, Nat. Photonics, 2011, 5, 406-410.

6 M. C. Gather and S. H. Yun, Opt. Lett., 2011, 36, 3299-3301.

7 Q. Chen, X. Zhang, Y. Sun, M. Ritt, S. Sivaramakrishnan and X. Fan, Lab Chip, 2013, 13, 2679-2681.
$8 \mathrm{X} . \mathrm{Wu}, \mathrm{Q}$. Chen, Y. Sun and X. Fan, Appl. Phys. Lett., 2013, 102, 203706.

9 S. Nizamoglu, M. C. Gather and S. H. Yun, Adv. Mater., 2013, 25, 5943-5947.

10 Y. Sun and X. Fan, Anal. Bioanal. Chem., 2011, 399, 205-211.

11 A. Kiraz, A. Sennaroglu, S. Doganay, M. A. Dundar, A. Kurt, H. Kalaycioglu and A. L. Demirel, Opt. Commun., 2007, 276, 145-148.

12 T. Nagai, K. Ibata, E. S. Park, M. Kubota, K. Mikoshiba and A. Miyawaki, Nat. Biotechnol., 2002, 20, 87-90.

13 T. Nagai, S. Yamada, T. Tominaga, M. Ichikawa and A. Miyawaki, Proc. Natl. Acad. Sci. U. S. A., 2004, 101, 10554-10559.

14 J. Yu, J. Xiao, X. Ren, K. Lao and X. S. Xie, Science, 2006, 311, 1600-1603.

15 M. Drobizhev, N. S. Makarov, S. E. Tillo, T. E. Hughes and A. Rebane, Nat. Methods, 2011, 8, 393-399.

16 A. Miyawaki, Annu. Rev. Biochem., 2011, 80, 357-373.

17 N. C. Shaner, P. A. Steinbach and R. Y. Tsien, Nat. Methods, 2005, 2, 905-909.

18 G. J. Kremers, S. G. Gilbert, P. J. Cranfill, M. W. Davidson and D. W. Piston, J. Cell Sci., 2011, 124, 157-160.

19 A. Kiraz, Y. Karadag and M. Muradoglu, Phys. Chem. Chem. Phys., 2008, 10, 6446-6454.

20 G. C. Righini, Y. Dumeige, P. Feron, M. Ferrari, G. N. Conti, D. Ristic and S. Soria, Riv. Nuovo Cimento Soc. Ital. Fis., 2011, 34, 435-488.

21 J. D. Eversole, H. B. Lin and A. J. Campillo, Appl. Opt., 1992, 31, 1982-1991.

22 M. Aas, A. Jonáš and A. Kiraz, Opt. Commun., 2013, 290, 183-187.

23 V. D. Ta, R. Chen and H. D. Sun, Sci. Rep., 2013, 3, 1362.

24 C. Eggeling, J. Widengren, R. Rigler and C. A. M. Seidel, Anal. Chem., 1998, 70, 2651-2659.

25 P. Sarkar, S. V. Koushik, S. S. Vogel, I. Gryczynski and Z. Gryczynski, J. Biomed. Opt., 2009, 14, 034047.

26 J. D. Eversole, H. B. Lin, C. D. Merritt and A. J. Campillo, Appl. Spectrosc., 1994, 48, 373-381.

27 C. Eggeling, A. Volkmer and C. A. M. Seidel, ChemPhysChem, 2005, 6, 791-804.

28 M. K. Daddysman and C. J. Fecko, Biophys. J., 2011, 101, 2294-2303.

29 C. L. Woldringh and N. Nanninga, J. Struct. Biol., 2006, 156, 273-283.

30 E. Özelci, M. Aas, A. Jonáš and A. Kiraz, Laser Phys. Lett., 2014, 11, 045802. 